\title{
Diseño de materiales multimedia para la formación de competencias informacionales
}

\author{
Design of multimedia materials for information literacy instruction
}

\author{
José Antonio SÁNCHEZ SUÁREZ \\ Biblioteca Universitaria de las Palmas de Gran Canaria, Edificio Central de la Biblioteca Universitaria. \\ Campus Universitario de Tafira s/n. 35017 Las Palmas de Gran Canaria, jsanchezs@pas.ulpgc.es
}

\begin{abstract}
Resumen
Los tutoriales multimedia se han convertido en objetos de aprendizaje esenciales en las políticas de formación en competencias informacionales, así como en el aprendizaje autónomo que se potencia en el Espacio Europeo de Enseñanza Superior. La creación de los materiales multimedia debe fundamentarse en una serie de principios con el objetivo de facilitar el proceso de aprendizaje de los estudiantes, por ello, se deben tener en consideración aspectos como los mecanismos de aprendizaje de la memoria, un estilo pedagógico o la interacción con los estudiantes. El diseño instruccional -ADDIE- tendría como objetivo planificar el desarrollo de una política institucional para la elaboración de materiales multimedia. La colaboración en los procesos de enseñanza es uno de los elementos claves en el EEES. Las bibliotecas deberemos establecer mecanismos para desarrollar procesos formativos en competencias informacionales, así como en la elaboración de materiales multimedia.
\end{abstract}

Palabras clave: Materiales multimedia. Tutoriales. Competencias informacionales. Bibliotecas universitarias. ADDIE.

\section{Introducción}

Las bibliotecas universitarias durante los últimos años han manifestado una preocupación constante por la formación de los usuarios en competencias informacionales. Los procesos de formación se han centrado en la elaboración de cursos, integración en asignaturas que en muchos casos se ven apoyados por objetos de aprendizaje como guías, blog o materiales multimedia. Los materiales multimedia juegan un papel fundamental en los procesos de formación, pues agilizan el proceso de aprendizaje, favorecen el trasvase de información y conocimiento, pero también porque ayuda a crear procesos mentales coherentes. El diseño de estos materiales deberían definirse a partir de una política de diseño instruccional que tuviera en consideración la adaptación de los mismos a un modelo pedagógico como puede ser, por ejemplo, el constructivismo. La función del diseño instruccional tiene como objetivo realizar un

\begin{abstract}
Multimedia materials have become essential learning objects in information literacy policy, specially for promoting autonomous learning, which is a key objective in the European Space for Higher Education. The creation of multimedia materials must be based on a series of principles with the aim of making the learning process easy for the students. Therefore, it must have considered aspects such as the mechanism of learning, the pedagogical style or the interaction with the student. Instructional design (ADDIE) would have as its main goal to plan the development of an institutional policy for the creation of multimedia materials. Collaboration is an essential element in the process of learning within the European Space for Higher Education. Libraries must jointly establish methods to cooperate in information literacy learning process and in creation of multimedia materials.
\end{abstract}

Keywords: Multimedia materials. Tutorials. Information literacy. Academic libraries. ADDIE.

estudio completo de las necesidades educativas de estos materiales, así como definir una política de elaboración de los mismos según las necesidades de la biblioteca.

La cooperación entre universidades es uno de los ejes recogidos en el Espacio Europeo de Enseñanza Superior que la biblioteca deberá asumir en los procesos de formación de competencias informacionales, lo cual puede manifestarse en la elaboración de una política de intercambio de materiales docentes o materiales multimedia, pues en la mayoría de los casos las bibliotecas compartimos recursos electrónicos.

Con el presente trabajo pretendemos, por un lado, estudiar desde un punto de vida psicopedagógico diferentes tipos de tutoriales realizados por las bibliotecas universitarias españolas. Por otro, analizar las ventajas de un sistema de diseño instruccional, como ADDIE o Polaris, en 
el establecimiento de una política de creación de tutoriales multimedia.

\section{Principios}

En el diseño de materiales multimedia debemos seguir una estructura y unos principios, tal como establece Mercè Gisbert Cervera, con el objetivo de facilitar el proceso de aprendizaje.

\subsection{Simplicidad}

Implicaría, por un lado, que el tutorial sea lo suficientemente ágil en su proceso de descarga para que el usuario pueda visualizarlo de forma continuada. Por ello, los procesos de streaming juegan un papel fundamental. En aquellos casos en los que este sistema no garantice una calidad suficiente de visualización, se podrá optar por dar al usuario la posibilidad de bajarse el fichero. La agilidad debe estar dirigida a no causar cansancio o aburrimiento en el usuario.

Por otro lado, la información que se proporciona en los tutoriales debe ser la adecuada, pues un exceso de información o que no esté acorde al nivel de aprendizaje puede dificultar al usuario el proceso de educativo. Sin embargo, tampoco debemos defraudar las expectativas de los usuarios, por lo que se hace necesario un análisis de las características de los diferentes tipos de usuarios de la biblioteca para poder adaptar el material multimedia a cada una de las necesidades. En algunos casos, será necesario profundizar en el contenido de los tutoriales, por lo que se hará necesario dividir la información en varios tutoriales, pues según las recomendaciones de Ruth Clark (2007), los tutoriales no deberán sobrepasar los 5 minutos.

\subsection{Didáctica}

El proceso de aprendizaje de nuestra memoria juega un papel fundamental en el momento de diseñar un tutorial multimedia. Dicho proceso depende de dos componentes esenciales: la memoria activa u operativa y la memoria de largo plazo.

La memoria activa será donde se desarrolle el proceso de aprendizaje, pero, por desgracia, tiene una capacidad muy limitada, por lo que el exceso de información provoca la disminución de su capacidad para llevar a cabo su función. Esta parte de la memoria se divide en varios subsistemas: un sistema supervisor y dos almacenes secundarios. El primero de ellos, lazo articulatorio, es el responsable del almacenamiento pasivo y de la información verbal, mientras que el segundo, agenda visoespacial, se encargará de almacenar la información visual o espacial. El proceso de aprendizaje se ve limitado, por tanto, cuando se realizan actividades de forma simultánea y dichas tareas requieren de un almacén secundario.

En el diseño de los materiales multimedia, según Rurk Clark (2007), para obtener un proceso adecuado del aprendizaje deberemos evitar el efecto de redundancia que se produce con la combinación de texto con sonido que puede provocar la saturación de la memoria. Por tanto, la combinación idónea para la elaboración de material multimedia será vídeo con audio, aunque en algunos casos deberemos utilizar una triple combinación vídeo, texto y audio para facilitar la accesibilidad de dichos materiales, aunque tendremos que darle al usuario la posibilidad de desactivar el texto de los subtítulos. Herramientas para crear tutoriales como Adobe Captivate, Camtasia Studio, así como Youtube y embebedores de streaming como JW Player o Kaltura permiten esta opción.

En las bibliotecas universitarias españolas, a diferencia de lo que sucede en las anglosajonas, la combinación predominante es la de vídeo y texto. Sírvanos de ejemplo, el Tutorial de Autoarchivo en RUA de la Universidad de Alicante o Cómo buscar en los formularios de búsqueda de SFX de la Universidad de la Carlos III.

La voz debe ser un elemento importante que debe ser capaz de atraer la atención del lector, por lo que la voz humana nos dará mejores resultados en el proceso de aprendizaje. Algunos de los tutoriales que nos hemos encontrado con voz son, por ejemplo, Polibuscador: Qué es y qué contiene de la Universidad Politécnica de Valencia o Acceda de la Universidad de Las Palmas de Gran Canaria, aunque destacaremos Cerca per autor de la Universitat Autònoma de Barcelona por el juego de voces que se realiza, que consiguen llamar la atención del usuario.

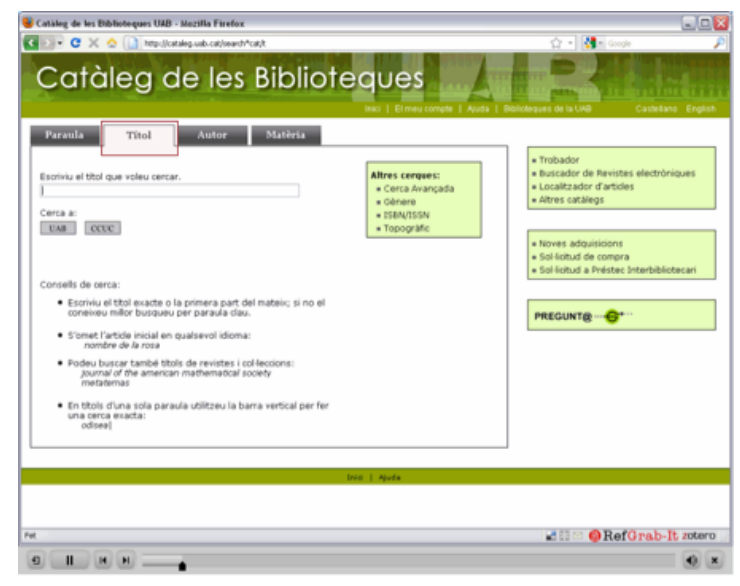

Figura 1. Imagen del tutorial Catàlogeg de les Biblioteques de la UAB 


\subsection{Dinamicidad}

Los materiales multimedia deben atraer la atención del usuario por lo que una de sus características es que deben ser dinámicos, así como ofrecer unos contenidos de calidad y un diseño imaginativo. El abuso de texto en los tutoriales multimedia, por ejemplo, reduce la agilidad de los mismos, pues debemos dejar un tiempo suficiente para que el usuario sea capaz de leerlo, lo cual es difícil de calcular, pues el ritmo de lectura es diferente según cada persona.

La mayoría de los tutoriales elaborados por las bibliotecas universitarias españolas se fundamentan en el screencast, sin embargo dentro del ámbito anglosajón nos encontramos ejemplos como Finding books de la Brown Library, donde se combina el screencast con personajes reales, utilizando además el humor como un elemento más para atraer la atención de usuario.

\subsection{Legibilidad}

La calidad de todos los elementos que constituyen el tutorial (vídeo, imágenes, sonido, etc.), así como la sincronización de los mismos favorece su legibilidad.

El desarrollo de los vídeos de High Definition (HD) con resolución $1280 \times 720$ ó $1920 \times 1080$ pixeles ha mejorado la calidad de los tutoriales alojados en Youtube. La Universitat Oberta de Catalunya trabaja con una calidad de 720HD. Nos puede servir de ejemplo Competències Informacionals - Vlex.

\subsection{Interacción}

La interacción con los usuarios en el proceso educativo es un elemento fundamental en modelos pedagógicos como el constructivismo social.

Algunos programas como Adobe Captivate potencian la interacción gracias a la posibilidad de establecer simulaciones. La interacción en los materiales multimedia, por ejemplo en las simulaciones, permite al usuario definir su propio ritmo de aprendizaje, basado en sus experiencias, lo que le permite crear sus propias construcciones mentales.

Por otro lado, el proceso de interacción también se establece permitiendo a los usuarios participar con sus opiniones o realizando preguntas que los bibliotecarios u otros usuarios podremos responder por el mismo medio, tal vez ese sea uno de los éxitos de Youtube. Desde el punto de vista de la biblioteca, nos permitiría conocer los puntos fuertes y puntos débiles de los tutoriales, lo que nos ayudaría a definir políticas de mejora sobre los mismos. La integración de los tutoriales en CMS como Drupal también permite a los usuarios realizar dichos comentarios como podemos ver en el vídeo Factor de impacto en JCR de la Biblioteca de la Universidad de Las Palmas de Gran Canaria. Se podría optar, igualmente, por el establecimiento de un formulario donde se permitiera expresar la opinión a los usuarios.

Este tipo de interacción permite a los usuarios a exponer sus experiencias, por lo que facilitaría la adquisición del conocimiento, pues según Vigotsky el aprendizaje es fruto de un acto social y no individual.

El usuario deberá tener la posibilidad de desplazarse por los diferentes elementos de forma autónoma, así como facilitar la integración de los diferentes elementos que componen el tutorial (vídeo, texto, imágenes, etc.).

El desarrollo de materiales multimedia favorece que el usuario puede definir su propio esquema de aprendizaje, al estar elaborado por nodos y enlaces organizados en estructuras semánticas. De esta manera, tutoriales elaborados con programas como Adobe Captivate o formato web facilitan que el estudiante defina su propio proceso de aprendizaje.

Tomás A. Pérez (2001), entre otros, analiza en Hipermedia, adaptación, constructivismo e instructivismo las ventajas de los objetos de aprendizaje basados en el hipertexto en el proceso educativo fundamentados en el constructivismo social, al potenciar entre otras cosas el autoaprendizaje.

\subsection{Flexibilidad}

Facilitar el acceso a los contenidos con el objetivo de que puedan ser consultados desde diferentes entornos. La posibilidad de embeber este tipo de materiales nos permite integrarlos en diferentes entornos, ya sean páginas web, LMS como Moodle, etc.; por ello, herramientas como la Videoteca de la Universitat Politècnica de Catalunya, que facilita a los usuarios el código embebedor en HTML para difundirlos en diferentes soportes según sus necesidades, lo cual — combinado con el acceso abierto- puede ser un elemento esencial para potenciar la rentabilidad de dichos materiales y la cooperación entre bibliotecas.

\section{Diseño instruccional}

Según D. R. Wegener (2006) el diseño instruccional está dirigido a definir los elemento claves como quién, qué, cuándo, dónde, por qué y 
cómo de un programa educativo. Por tanto, podemos decir que tiene como objetivo realizar un estudio completo que los elementos que se han de tener en consideración en un proceso $u$ objetivo de aprendizaje: planificar los objetivos, establecer las estrategias instruccionales, seleccionar y elaborar los objetos de aprendizaje y la evaluación.

De los diferentes modelos de diseño intruccional, ADDIE tal vez sea el más adecuado para definir un programa de competencias informacionales o el diseño de materiales multimedia. El acrónimo ADDIE define claramente las diferentes etapas que lo constituyen: análisis, diseño, desarrollo, implementación y evaluación. La evaluación será uno de los elementos que se aplique a todos los procesos de este modelo, por lo que se han de establecer los indicadores correspondientes (Branch, 2009). La combinación de modelos ADDIE con el diagrama Gantt nos permitirá una gestión y planificación perfecta de cualquier proyecto (tabla I).

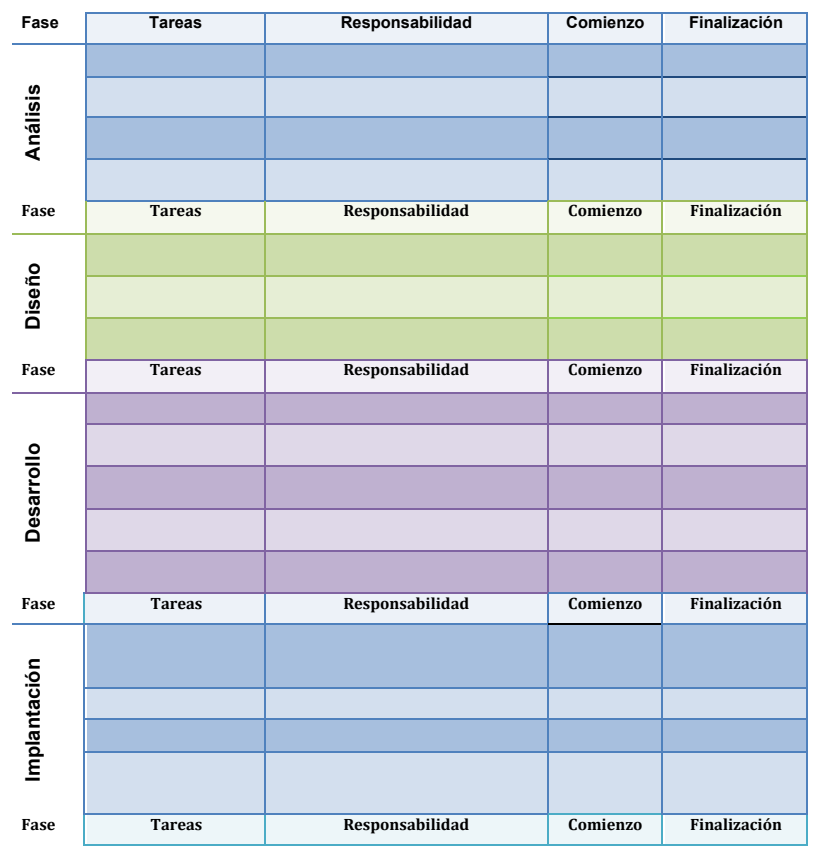

Tabla I. Modelo de planificación

\subsection{Análisis}

La fase de análisis implica el estudio de aquellos elementos necesarios para la elaboración del proyecto.

Analizar los conocimientos, habilidades y capacidades informacionales requeridas por los usuarios. Uno de los elementos claves para conocer las necesidades formativas en competencias informacionales entre nuestros estudiantes se fundamenta en la colaboración con los docentes, quienes nos pueden asesorar o guiar sobre aquellos materiales multimedia que son necesarios según los diferentes niveles educativos.

El estudio de los recursos necesarios para llevar a cabo el proyecto se reflejará en un análisis de los diferentes programas informáticos que puedan servirnos para adaptarnos a nuestras políticas formativas. Por ello, deberemos analizar previamente, por ejemplo, la compatibilidad de nuestros programas LMS (Moodle, Sakai, etc), CMS (Drupal, Joomla, etc.) o páginas web con los diferentes formatos que nos ofrecen los programas para la elaboración multimedia.

Se sugiere la adopción de un estilo pedagógico como el constructivismo social que se fundamente en aspectos como que el aprendizaje se construye a partir de la propia experiencia, por lo que aquellos tutoriales que presenten la posibilidad de realizar simulaciones potenciarían el proceso de aprendizaje.

Los tutoriales multimedia permitirán que sea el propio estudiante el que defina su proceso de aprendizaje de acuerdo con sus hábitos y expectativas.

Por último, el constructivismo social se fundamenta en la relación entre docentes (bibliotecarios) y estudiantes (usuarios), por lo que facilitar la interacción con los mismos a través de la posibilidad de hacer comentarios juega un papel fundamental, potenciando además la relación entre los propios estudiantes, que pueden intercambiar ideas a través de los mismos.

Por último, será necesario establecer una política de formación de personal sobre las diferentes herramientas necesarias para el desarrollo de los materiales y su integración en el proceso formativo.

\subsection{Diseño}

El diseño supone poner en práctica una serie de actividades complejas destinadas a facilitar el proceso formativo. El diseño de materiales multimedia, según Jesús Valverde Becorroso, debe fundamentarse en cuestiones como qué, a quién y para qué: el qué determinará el contenido del material, el a quién condicionará el desarrollo del contenido - pues dependiendo del tipo de usuario al que vaya dirigido se determinará por ejemplo, el nivel de profundidad-, mientras que el para qué dependerá de las finalidades educativas - no será lo mismo que se trate de un tutorial para la formación de unas jornadas de acogida que para la formación especializada de un máster o doctorado. 
El proceso de diseño tiene como función establecer las estrategias formativas, definir los objetivos formativos de los materiales multimedia, así crear un plan para la elaboración de guiones de los materiales multimedia.

El guión o guía didáctica tiene como objetivo, tal como indica Elena Galán Fajardo, planificar los detalles necesarios para la elaboración del material multimedia. Por ello, recogerá elementos como el título, autores, destinatarios, herramientas, escenas, etc. Así pués, debemos entender la existencia de diferentes tipo de elementos que componen el guión o la existencia de diferentes tipos de guiones (tabla II).

- Contenido: Define las diferentes escenas de manera jerarquizada.

- Narrativo: Tendrá como objetivo organizar la información desarrolladas en las diferentes escenas, así como definir los textos que emplearemos en el material que vamos a desarrollar.

- Icónico: Se definirán las imágenes, gráficos, figuras, etc., que se utilizaran en las diferentes escenas.

- Sonido: Se deberá desarrollar de forma sincrónica con el narrativo y anotarán aquellos que se incluyan en el tutorial.

- Técnico: Se identificarán todos aquellos programas o plataformas que se utilizarán para la elaboración o ubicación del material, así como los formatos de presentación y requerimientos técnicos para su visualización.

\begin{tabular}{ll}
\hline Título & Destinatarios \\
\hline Autor/es: & Objetivos \\
\hline Resumen & Herramientas \\
\hline Versión & $\begin{array}{l}\text { Requisitos informáticos } \\
\text { para el usuario }\end{array}$ \\
\hline Observaciones & Ubicación \\
\hline & \\
\hline $\begin{array}{l}\text { Descripción de la } \\
\text { escena }\end{array}$ & Imágenes Texto Audio \\
\hline
\end{tabular}

Tabla II. Datos básicos del guións

\subsection{Desarrollo}

En la fase de desarrollo pondremos en práctica con los programas que hayamos elegido la elaboración de los tutoriales multimedia y los integraremos en plataformas, web, repositorios, etc., que consideremos adecuado para el proceso formativo. Sin embargo, antes de la implantación definitiva será necesario establecer un período de prueba con un limitado número de estudiantes o personal de la biblioteca para poder evaluar si dichos materiales cumplen con los objetivos establecidos, así como para definir posibles mejoras de los mismos.

\subsection{Implementación}

La fase de implantación supone la puesta en marcha del proyecto, lo que se traduce en que los usuarios pueden proceder al uso de los mismos para el proceso formativo.

\subsection{Evaluación}

La evaluación la debemos plantear desde dos direcciones. Por un lado, un análisis del proceso de elaboración de los materiales multimedia que analice los puntos fuertes y las debilidades de las diferentes fases del modelo ADDIE, lo cual nos permite establecer las mejoras para futuros proyectos. Por otro, debemos evaluar la calidad de materiales multimedia desde diferentes puntos de vista relacionados con el diseño tecnológico y pedagógico, que según César Coll y Anna Engel (2008) debe comprender los siguientes elementos:

- Identificación del material: El título servirá para que usuario identifique el contenido del documento, lo que le permitirá saber si contiene la información que necesita o no. Los datos relacionados con los autores (personal o institucional) permiten a los usuarios ponerse en contacto con ellos en caso de duda. Los datos técnicos harán referencia a las necesidades de programas para la visualización de los tutoriales que los usuarios necesitan conocer. Además, estos datos permiten a las bibliotecas usarlos como metadatos para identificarlos en los repositorios destinados a los objetos de aprendizaje.

- Accesibilidad: Los indicadores deberán estar destinados a analizar la calidad de recepción de los diferentes elementos que constituyen el material multimedia, así como la navegación por el mismo. Por ello, este elemento estará relacionado con los principios de simplicidad y hipertextualidad.

- Calidad técnica de los materiales: Se analizarán los diferentes elementes utilizados para el desarrollo del tutorial (imágenes, textos, sonidos, etc.), así como la sincronización entre ellos. 
- Objetivos y contenidos: Los indicadores estarán relacionados con que los contenidos están vinculados con los objetivos establecidos en proceso de aprendizaje, así como con la complejidad de los mismos.

- Presentación, organización y secuenciación de los contenidos: Se analiza la organización correcta de los contenidos, así como la visibilidad y ritmo de los mismos.

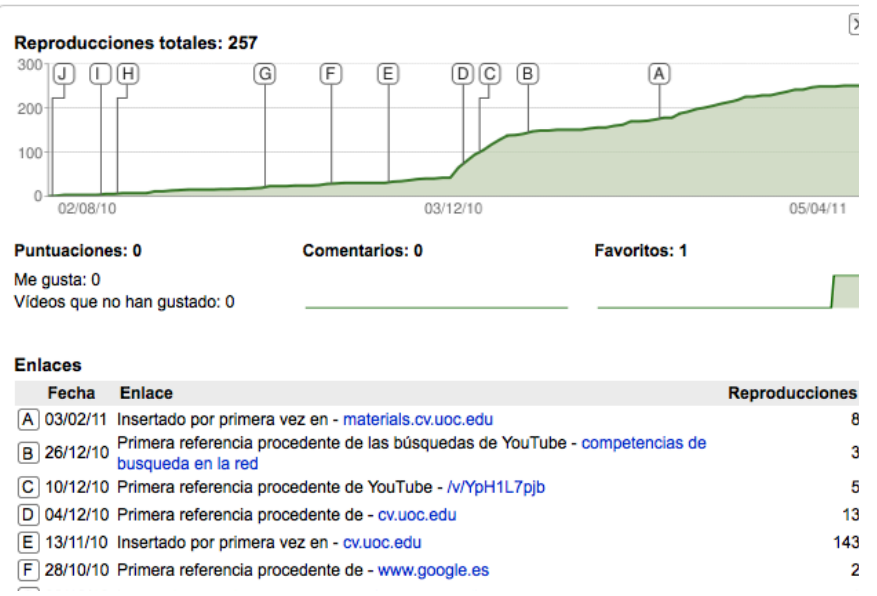

Figura 2. Estadística de Youtube del tutorial El catálogo de la UOC

Por otro lado, sería interesante cuantificar la visualización de dichos materiales por los usuarios. Herramientas como Youtube nos dan esos datos, pero Google Analytics puede ser un gran aliado para estudiar el uso de los tutoriales elaborados con Camtasia y Adobe Captivate, como lo demuestra Betty (2009) en el estudio elaborado en la Regis Library.

\section{La colaboración en la elaboración de materiales multimedia}

El Espacio Europeo de Enseñanza Superior tiene entre sus ejes fundamentales la colaboración en las tareas docentes. Esto que no hace referencia únicamente al intercambio temporal de docentes, sino que implicaría la colaboración en la elaboración de unidades didácticas, así como en el intercambio o elaboración de forma conjunta de objetos de aprendizaje.

La colaboración, según Vygotsky (2001), es el proceso de creación compartida por dos o más personas con el objetivo de crear un proceso, un producto o un evento a través de la interacción y el intercambio de conocimientos.

En el ámbito anglosajón es frecuente el desarrollo de repositorios de tutoriales con el objetivo de facilitar el intercambio de los mismos entre bibliotecas. Es el caso por ejemplo de LOLA, Merlot y Ariadne.

La creación de repositorios que permitan el intercambio de objetos de aprendizaje, y por lo tanto materiales multimedia, en acceso abierto debería ser uno de los proyectos que las bibliotecas universitarias deberían plantearse en sus políticas de alfabetización información. Por otro, lado, la apuesta de la mayoría de las bibliotecas por la elaboración de materiales en formato vídeo podría permitir la creación de proyectos como Youtube Edu, pero que recogiera estos materiales de forma organizada, ya fuese por nombre de recurso o por biblioteca.

En España, sin embargo, las escasas políticas de colaboración de estos materiales las hemos localizado en el Grupo de REBIUN sobre Alfabetización Informacional con la elaboración de los tutoriales Competencias informáticas e informacionales en un mundo digital y Competencias informáticas e informacionales: ¿qué papel juegan las universidades?

La integración de las competencias informacionales en los procesos formativos universitarios como una función educativa de la biblioteca universitaria, debería obligar a las mismas a definir políticas de colaboración para el desarrollo de unidades didácticas, materiales multimedia, etc.

Las bibliotecas universitarias tienen experiencias en procesos de colaboración. Sin embargo son escasas las experiencias en este campo de los procesos formativos, aunque cabe destacar el Taller UNESCO de formación de formadores en alfabetización informacional.

La mayoría de las bibliotecas universitarias compartimos los mismos recursos electrónicos, por lo que los cursos virtuales bajo plataformas de educación virtual pueden ser intercambiados entre las bibliotecas, así como los tutoriales multimedia, e incluso establecer criterios y herramientas similares a Libguides para trabajar de forma conjunta en la elaboración de los mismos.

\section{Conclusiones}

La creación de materiales multimedia se ha convertido en esencial para la formación en competencias informacionales, lo que conlleva un esfuerzo por parte de las bibliotecas universitarias para que los mismos sean desarrollados a partir de criterios pedagógicos que faciliten el proceso de aprendizaje de los estudiantes.

La interacción con los usuarios - ya sea a través de materiales multimedia que sean plantea- 
dos como simulaciones, o por medio de la opinión e intercambio de ideas con los usuarios o entre ellos mismos- debe ser entendido como un elemento más en el proceso de aprendizaje.

La creación de un proyecto para el desarrollo de materiales multimedia debe fundamentarse en un proceso de diseño instruccional, con el objetivo de que se analicen las necesidades de los usuarios y que defina, entre otros aspectos, las necesidades formativas del personal, estudie los programas más adecuados según las necesidades de integración en diferentes plataformas, etc. Por ello, modelos de diseño instruccional como ADDIE o Polaris deben ser tenidos en consideración por parte de las bibliotecas.

La definición de políticas de colaboración entre las bibliotecas universitarias para la formación en colaboraciones informacionales será uno de los ejes fundamentales a tener en consideración en un futuro cercano, por lo cual será necesario establecer criterios para coordinar el desarrollo de unidades didácticas, materiales multimedia, etc.

El desarrollo de las herramientas de las web 2.0, así como plataformas de educación virtual, potencia las posibilidades de colaboración en competencias informacionales entre bibliotecas.

\section{Referencias}

Betty, P. (2009). Assessing homegrown library collections: Using google analytics to track use of screencasts and flash-based learning objects Journal of Electronic Resources Librarianship, 21(1), 75-92. http://dx.doi.org/ 10.1080/19411260902858631 (23-12-2010).

Brank, Robert Maribe (2009). Instructional Design: The ADDIE Approach. London: Springer. 2009

Clark, Ruth (2007). Aprovechamiento de los recursos multimedia para el aprendizaje. Adobe http://es.scribd.com/ doc/27273987/Captivate-Leveraging-Multimedia (21-112010).

Coll, César ; Engel, Anna (2008). La calidad de los materiales educativos multimedia: dimensiones, indicadores y pautas para su análisis y valoración. // Barberá, E.; Mauri, T.; Onrubia, J. (Eds.). Cómo valorar la calidad de la enseñanza basada en las TIC: pautas e instrumentos de análisis. Barcelona: Graó. 147-201.

Galán Fajardo, Elena (2006). El guión didáctico para materiales multimedia. // Espéculo: Revista de estudios literarios. 34.http://www.ucm.es/info/especulo/numero34/guio nmu.html

Gisbert Cervera, Mercé et al. (2005?). Conceptualización de materiales multimedia. Universitat Oberta de Catalunya.

León de Viloria, Chilina (2001). Lev Vygotsky: sus aportaciones para el siglo XXI. Caracas: UCAB.

Pérez, Tomás A. (2001). Hipermedia, adaptación, constructivismo e instructivismo. // Revista Iberioamericana de Inteligencia Artificial. 12, 29-38. http://redalyc.uaemex. $\mathrm{mx} / \mathrm{src} /$ inicio/ArtPdfRed.jsp?iCve=92551204 (1-7-2011)

Valverde Berrocoso, Jesús (2009?). Diseño y elaboración de materiales didácticos multimedia. http://www.slideshare. net/jevabe/diseo-y-elaboracin-de-materiales-didcticos-m ultimedia (12-04-2011)

Wegener, Debby R. (2006). Training Library Patrons the ADDIE Way (Information Professional). Oxford: Chandos Publishing. 2006. 
